\title{
A long term generation expansion planning model using system dynamics - Case study using data from the Portuguese/Spanish generation system
}

\author{
Adelino J.C. Pereira ${ }^{a}$, João Tomé Saraiva ${ }^{b, *}$ \\ a Departamento de Engenharia Electrotécnica, Instituto Superior de Engenharia de Coimbra, Instituto Politécnico de Coimbra, Rua Pedro Nunes, 3030 -199 Coimbra, Portugal

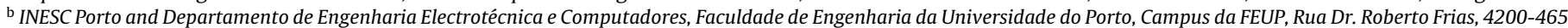 \\ Porto, Portugal
}

\section{A R T I C L E I N F O}

\section{Article history:}

Received 20 June 2012

Received in revised form 12 October 2012

Accepted 4 December 2012

\section{Keywords:}

Generation expansion planning

Long term analysis

System dynamics

Sensitivity analysis

Electricity markets

\begin{abstract}
A B S T R A C T
This paper describes a long term generation expansion model that uses system dynamics to capture the interrelations between different variables and parameters. Using this model, it is possible to estimate the long term evolution of the demand and of the electricity price that are then used by generation agents to prepare individual expansion plans. These plans are submitted to a coordination analysis to check some global indicators, as the reserve margin and the LOLE. The developed approach is illustrated using a realistic generation system based on the Portuguese/Spanish system with an installed capacity of nearly 120 GW and an yearly demand of 312 TWh in 2010. Large investments were directed in the last 20 years to the Iberian generation system both regarding traditional technologies and dispersed generation (namely wind parks and solar systems). Today, the excess of installed capacity together with the demand reduction poses a number of questions that should be addressed carefully namely to investigate the impact of several options. The planning exercise aims at identifying the most adequate expansion plans in view of the increased renewable generation (namely wind parks). For illustration purposes, we also conducted a sensitivity analysis to evaluate the impact of increasing the installed capacity in wind parks, of internalizing $\mathrm{CO} 2$ emission costs and of incorporating a capacity payment. These analyses are relevant in order to get more insight on the possible long term evolution of the system and to allow generation companies to take more sounded decisions.
\end{abstract}

(C) 2012 Elsevier B.V. All rights reserved.

\section{Introduction}

Power systems restructuring introduced new challenges both in operation and expansion planning activities. In particular, the generation expansion planning, GEP, problem was already a matter of concern for vertically integrated utilities and it was typically addressed in a combined way with transmission expansion planning. This was a complex problem given the discrete nature of possible investments, as well as their capital intensive nature and their one-step and irreversible characters, in the sense that once a decision was taken it could hardly be reversed. Finally, these were typically long pay back investments that required a long term analysis. However, before restructuring the risk of these investments was much more reduced than now given that the introduction of market mechanisms imposed a shorter term accent, the demand is now more uncertain and the increasing presence of renewables (namely using volatile resources as wind and solar radiation) originates new challenges not only for operation but also for long term

\footnotetext{
* Corresponding author. Tel.: +351 22 2094230; fax: +35122 2094150.

E-mail addresses: ajcp@isec.pt (A.J.C. Pereira), jsaraiva@fe.up.pt (J.T. Saraiva).
}

activities. The restructuring lead to the introduction of competitive mechanisms in generation and retailing while usually keeping transmission and distribution wiring as regulated activities. In generation there are now several companies owning assets and competing to supply the demand so that the profit of a particular agent is affected by the demand and fuel prices evolution, by the incentives given to renewable stations and also by the decisions taken by other companies. As a result, the traditional multiyear mixed integer GEP problem is now much more than in the past influenced by uncertainties affecting several parameters that should be internalized in the decision process. Finally, there are interdependencies that should be adequately modeled to capture the real nature of the problem. In fact, investment decisions are driven by the expected profit along the horizon, which is influenced by the evolution of the demand and by the market prices. However, these two elements are interdependent to some extent and also depend on the evolution of fuel costs. This suggests the adoption of a systematic modeling tool to adequately represent all these interactions.

The objective of a GEP problem is to identify the most adequate investment schedule of generation plants together with their sitting and technology to supply the demand considering its possible 
evolution along the planning period while enforcing some reliability constraints. The GEP problem was usually addressed in an integrated way with transmission expansion planning [1,2]. Reference [1] details an approach using Stochastic Programming to model several uncertain parameters and [2] describes a mixed integer optimization problem that uses the DC model of the transmission system. References [2-4] use Benders decomposition and $[5,6]$ describe multi-objective approaches considering investment, operation and transmission costs, environmental impacts and the risk of getting a plan very much exposed to uncertainties.

More recently, metaheuristics started to be used to solve the GEP problem, taking into account its combinatorial nature. In this scope, [7-10] report the use of genetic algorithms, simulated annealing, expert systems, ant colonies and particle swarm algorithms as well as combinations of these techniques [10]. These models minimize operation plus investment costs subjected to a number of constraints, for instance regarding the maximum amount of investment that can be accommodated in each planning period. Metaheuristics, both using a solution in each iteration or population based, characterize the investment plans using an evaluation function that combines the optimization function of the original problem plus penalties on the violated constraints.

The advent of restructuring created new concerns namely whether expansion plans prepared by individual generation agents are capable of ensuring the security of supply on the long term. In this scope, [11] describes a two step approach in which individual agents prepare expansion plans maximizing their profit that are then submitted to an upper level check to investigate the quality of the global schedule, namely computing LOLP and the reserve margin along the horizon. On the other hand, [12] compares centralized and liberalized GEP models formulating an optimization problem to maximize the profit of the investors. This problem is solved using dynamic stochastic programming together with discrete Markov Chains to model the demand uncertainty.

Finally, in recent years system dynamics started to be applied to the GEP problem. System dynamics [13] was created by Jay Forrester in the 60s and several applications to power systems are reported in [14]. Regarding the GEP problem, [15,16] report long term electricity market models and [17] describes the application of these concepts to the generation system of New Zealand.

Recognizing the complexity of the GEP problem, the authors developed an initial approach to the TEP problem in which the demand and the electricity price evolution were obtained using a Cournot model [18]. This approach was then enhanced in [19] in which the long term evolution of the electricity market was modeled using system dynamics. In [19] it is used a generation system that mirrored the main characteristics of the Portuguese generation system scaled by $50 \%$ to illustrate the approach.

Apart from describing the main characteristics and blocks of the long term dynamic model as well as the profit maximization problems to be solved by each generation agent to schedule new generation investments, we are now applying this approach to a larger system that incorporates the main features of the Portuguese/Spanish generation system having a total installed capacity of nearly $120 \mathrm{GW}$ and an yearly demand of about $312 \mathrm{TWh}$ in 2010. It is important to analyze both systems in a global way given the common electricity market established between the two countries and the increasing interconnection capacity that strongly contributed to reduce congestions in the transmission lines between them. The main motivation for using the Portuguese/Spanish generation system comes from the challenges that the Iberian countries face in the next years. In fact, in recent years a conjunction of factors occurred namely regarding the large amount of investments that were directed to new generation stations in the last 20 years, the rapid increase of dispersed generation (including renewable technologies as wind parks and solar systems, as well

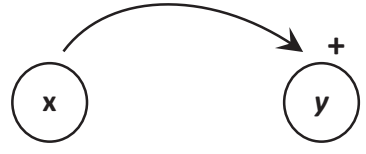

a. positive cause effect relation

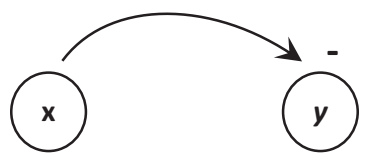

b. negative cause effect relation.
Fig. 1. Casual diagrams illustrating a positive relation in the left and a negative dependency in the right side.

as non renewable cogeneration systems) and the recent decrease of the demand due to the on-going economic crisis. All these situations strongly suggested that the policies followed in the last 20 years should be looked closely using long term approaches to more adequately evaluate the possible evolution of the system. On the other hand, as a result of the massive introduction of dispersed generation, the liquid demand available to be supplied by traditional thermal technologies is now much more reduced. In this scope, it is not rare that in winter wet valley periods the generation from wind parks and hydro stations becomes enough to supply the demand in Portugal, so that the output of traditional thermal stations comes to zero as well as the market price. As a consequence, the number of yearly operation hours of several CCGT stations is much reduced and so generation companies are now complaining that their revenues are not enough to pay these stations. As a result, a capacity payment was awarded to some Portuguese stations in 2009 but it was recently much reduced by the current government. This corresponds to an important issue that also deserves attention at the Iberian level. In order to address this issue, the GEP model described in [19] now incorporates a capacity payment so that we can perform sensitivity studies in order to adequately calibrate the value of this payment, for instance as a way to guarantee a minimum return rate for some less used thermal stations.

Considering these ideas, Section 2 provides an overview on system dynamics and Section 3 describes the overall adopted solution approach. Section 4 details the investment planning problems incorporating the mentioned capacity payment and Section 5 describes the long term dynamic model of the electricity market. Section 6 illustrates this approach with a case study using data taken from the Portuguese/Spanish generation system in the scope of the common electricity market established by the two countries since 2007 and Section 7 draws the most relevant conclusions.

\section{Overview on system dynamics}

System dynamics is a modeling tool particularly suited to represent long term problems that involve a large number of variables and parameters as well as loops and inter dependencies. In brief, adopting system dynamics to model a problem implies developing an in-depth understanding of the system to identify its boundaries, the time horizon and the most relevant variables, parameters and inter dependencies. Then, it is developed an initial dynamic model using standard basic models that will be briefly detailed below. The developed model is then translated into a mathematical formulation, that typically includes differential equations. Finally, using this formulation, the model is validated after which more involving studies and simulations can be developed.

The relations and dependencies between variables are represented using a number of standard sub-models. Figs. 1 and 2

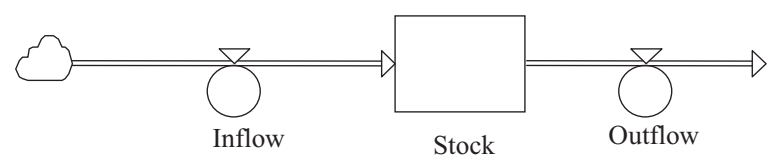

Fig. 2. Diagram of stocks and flows. 
illustrate positive and negative casual diagrams and the diagram of stocks and flows. As the name suggests, a positive loop represents a positive dependency between $x$ and $y$, in the sense that if $x$ increases then $y$ also increases, while a negative loop represents a negative relation between $x$ and $y$.

Diagrams of stocks and flows are intensively used in system dynamics. Stocks characterize the state of the system and based on them we can model decisions and actions. They represent the memory of the system and can be used to model delays and to decouple inflows from outflows. The flows represent the activity of the system and are used to establish relations between the stocks. The diagram of Fig. 2 is translated by (1) so that after modeling the system using these elementary models, we can translate the global casual diagram into a mathematical formulation.

$\frac{\partial \operatorname{Stock}(t)}{\partial t}=\operatorname{Input} \operatorname{Flow}(t)-$ Output Flow $(t)$

\section{Structure of the long-term expansion planning problem}

The long term evolution of the power system was modeled by the global casual diagram displayed in Fig. 3. Going along this loop clockwise, the decommissioning plans affect negatively the installed capacity. On the contrary, new additions have a positive impact in the reserves that, on the other hand, are negatively influenced by an increase of the electricity demand. The evolution of the demand together with information about the evolution of reserves will then be used to estimate the long term evolution of the electricity price that is also affected by the long term evolution of fuel prices. The profits of the generation companies are determined by the level of electricity prices as well as by the expected maintenance and investment costs. Finally, if reserves decline and electricity prices increase, it is transmitted a signal to build new power stations so that generation agents prepare expansion plans to maximize their profits along the horizon, using estimates for the demand and the electricity price evolution. This means that each generation agent solves its own profit maximization problem to select and schedule new power station investments. The set of new stations, that is, the long term planned evolution of the generation system, is then checked evaluating some reliability indices (as the LOLE and the reserve margin). This step can also involve checking if the installed capacity in some technologies does not exceed maximum values if specified in national energy strategic plans. Accordingly, departing from the existing generation mix, the dynamic model is used to estimate the evolution of the demand, of the electricity price and of the capacity factor of each technology, expressing the percentage of hours during which, in average, each technology is used. These outputs are then used by generation companies to prepare their own expansion plans. Using these plans, the dynamic model is run again to update the mentioned estimates thus defining an iterative procedure. In Section 4 we will detail the mentioned investment problems and Section 5 describes the main blocks of the dynamic model to get the long term evolution of the power system.

\section{Identification of investment plans}

\subsection{Individual investment problems}

Admitting that the evolution of the demand, of the electricity price and of the capacity factor of each technology along the horizon are known, each generation company, Genco, solves an optimization problem to maximize its own profit considering the costs and the revenue from selling electricity. When doing this, a Genco can decide investing in new power stations considering a number of candidate technologies characterized by their possible installed capacity, investment and operation and maintenance costs. This investment planning problem can be modeled by the mixed integer optimization formulation given by (2-8) in which the decision variables $X_{t}^{i, j}$ represent the new capacity of technology $j$ to be commissioned in stage $t$ by Genco $i$.

$$
\begin{aligned}
\max z= & \sum_{t=1}^{T}\left[\sum_{j=1}^{M} \pi^{t} \cdot C C_{t}^{i j} \cdot \Delta t \cdot \alpha_{t}^{i j}+\sum_{j=1}^{M} P_{c a p}^{j} \cdot C C_{t}^{i j} \cdot\left(1-\alpha_{t}^{i j}\right)\right. \\
& \left.-\sum_{j=1}^{M} C i n v_{t}^{j} \cdot X_{t}^{i j}-\sum_{j=1}^{M} \operatorname{Cop}_{t}^{j} \cdot C C_{t}^{i j} \cdot \Delta t \cdot \alpha_{t}^{i j}\right]
\end{aligned}
$$

$\operatorname{subj} X_{t}^{i j} \leq M I C_{t}^{i j}$

$\sum_{j=1}^{M} X_{t}^{i j} \leq M I C_{t}^{i}$

$\sum_{j=1}^{M} X_{t}^{i j} \cdot C i n v_{t}^{j} \leq M X I N V_{t}^{i}$

$\sum_{t=1}^{T} \sum_{j=1}^{M} X_{t}^{i j} \cdot C i n v_{t}^{j} \leq M X I N V^{i}$

$C C_{t}^{i j}=C C_{t-1}^{i j}+X_{t}^{i j}$

$t=1, \ldots, T ; \quad i=1, \ldots, N ; \quad j=1, \ldots, M$

In this formulation $T$ is the number of stages in the planning horizon; $t$ is the stage in the planning horizon; $\Delta t$ is the duration of each stage in the planning horizon; $M$ is the number of candidate technologies; $j$ is the type of candidate expansion technology; $i$ is the index associated to a particular GENCO; $\pi^{t}$ is the electricity price in stage $t(€ / \mathrm{MWh}) ; P_{\text {cap }}^{j}$ is the capacity payment set for technology $j$ $(€ / \mathrm{MW}) ; \alpha_{t}^{i j}$ is the capacity factor in stage $t$ for GENCO $i$ and technology $j$ (percentage); $C i n v_{t}^{j}$ is the investment cost for technology $j$ at stage $t(€ / \mathrm{MW})$; $\operatorname{Cop}_{t}^{j}$ is the variable operation and maintenance cost for technology $j$ at stage $t(€ / \mathrm{MWh}) ; C C_{t}^{i j}$ is the cumulative capacity installed in stage $t$ for GENCO $i(\mathrm{MW}) ; X_{t}^{i, j}$ is the capacity addition of technology $j$ in stage $t$ by GENCO $i(\mathrm{MW})$; $M I C_{t}^{i j}$ is the upper bound set for the installed capacity of technology $j$ in stage $t$ by GENCO $i(\mathrm{MW})$; $M I C_{t}^{i}$ is the maximum capacity installed in stage $t$ by GENCO $i(\mathrm{MW}) ; M X I N V_{t}^{i}$ is the maximum value specified for the capital investment of GENCO $i$ at stage $t(€)$; MXINV $i$ is the maximum investment of GENCO $i$ along the horizon $T(€)$.

The objective function (2) contains four terms. The first one represents the revenues obtained by the generation agent $i$, Genco $i$ along the horizon $T$ from selling electricity at the market price in each period $t$ using the installed capacity and the capacity factor $\alpha_{t}^{i, j}$ for each technology. The second term models a capacity payment eventually set by administrative or regulatory agencies to remunerate generation stations during the periods they are not in operation. As mentioned in Section 1, such terms already exist in some countries and are under discussion in several others to cope with the reduction of the operation hours of several thermal stations due, for instance, to the increase of the injections coming from dispersed generation, namely wind parks and solar systems. The third term represents the investment cost in each period of the horizon for each technology and the fourth one corresponds to the operation and maintenance costs along the horizon. It should be mentioned that when computing the value of this objective 


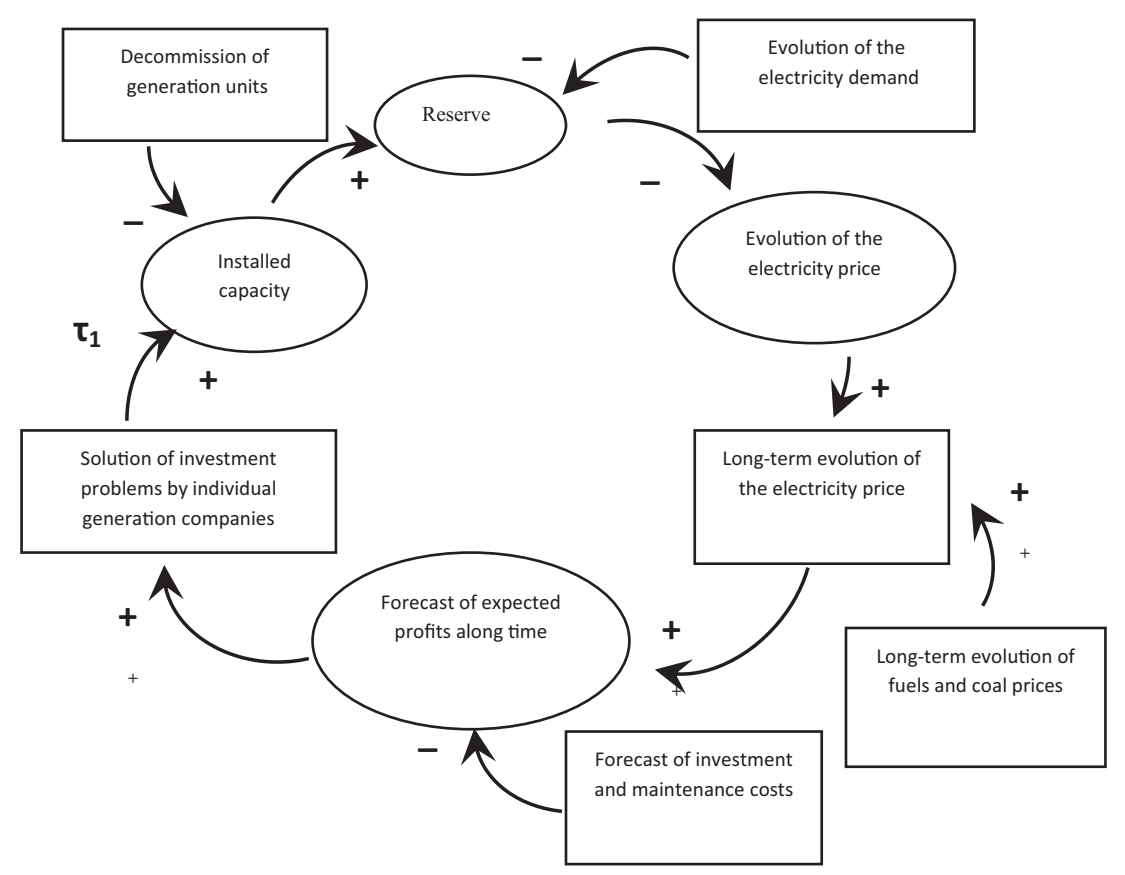

Fig. 3. Casual diagram modeling the long term power system evolution.

function the revenues and costs along the planning horizon are brought to the initial period using an adequate actualization rate.

This objective function is subjected to constraints that limit the new capacity to be added in each period $t$ for each technology $j$ for $\mathrm{Genco}_{i}(3)$, that enforces the limit on the new capacity additions of $\mathrm{Genco}_{i}$ in each period $t$ of the horizon (4) and that limits investment costs either for each period $t(5)$ or for the entire horizon $T$ (6) reflecting financial limitations of each agent. Finally, (7) is used to update the global installed capacity of $\mathrm{Genco}_{i}$ as the planning horizon develops.

This is a mixed integer problem that was solved using a genetic algorithm as briefly detailed below:

(i) Initialization - the initial population is randomly sampled considering the available capacities that it is possible to install of each technology. Each individual is obtained sampling the number of stations of each technology $j$ to start operation in each period $t$ in $T$.

(ii) Evaluation - each individual corresponds to a possible expansion plan. The associated profit is given by (2) using the evolution of the electricity prices and of the capacity factors provided by the long term analysis. Constraints (3)-(7) are also checked and if violated then the fitness function is given by (2) minus penalties over the violated constraints.

(iii) Convergence check - the convergence is monitored checking the average value and the standard deviation of the fitness function of the individuals in the current population. The algorithm converges if the average value of the fitness function is sufficiently stable for a pre specified number of iterations, the standard deviation is smaller than a specified threshold and the fitness function of the best individual (the one having the largest fitness value) did not change more than a specified threshold for a pre specified number of iterations. We also imposed a minimum number of iterations to be completed independently of the convergence checking.

(iv) Genetic operators - if the convergence criteria were not met, then the algorithm proceeds to a new iteration applying the selection, cross-over and mutation operators over the current population. Then the algorithm returns to step (ii) to analyze the individuals in the new population.

When this iterative process stops, the expansion plan of the generation company $i, \mathrm{Genco}_{i}$, will correspond to the individual in the final population that displays the largest profit.

\subsection{Coordination analysis}

As mentioned in Section 3, each generation agent should prepare its own expansion plan. This exercise can also be done by $\mathrm{Genco}_{i}$ that wants to develop its own expansion plan while admitting pre-specified commissioning schedules for all other agents. The expansion plan for $\mathrm{Genco}_{i}$ can then be tested against different additions of the other competitors to investigate its sensitivity to the decisions of other agents.

In any case, the generation system should guarantee the supply of the demand displaying an adequate security and reliability. To ensure that specified standards are met along the horizon we submit the set of developed partial plans to a coordination analysis that includes calculating:

- the loss of load expectancy measuring the number of hours along each year that the generation system may not be able to supply the demand. The yearly LOLE values are compared with a maximum set value and if some yearly LOLE exceeds the maximum, then the global planning exercise did not converge yet.

- The yearly value of the reserve margin, corresponding to the excess, in percentage, of the global installed capacity regarding the peak power. The yearly values of this margin should be bounded by a minimum value to contribute to ensure the security of supply and eventually by a maximum value to prevent an excessive installed capacity.

Finally, it is possible to limit the installed capacity owned by each generation company against the global capacity in the system as well limiting, for instance, the installed capacity for a particular technology in the scope of public national energy policies. 


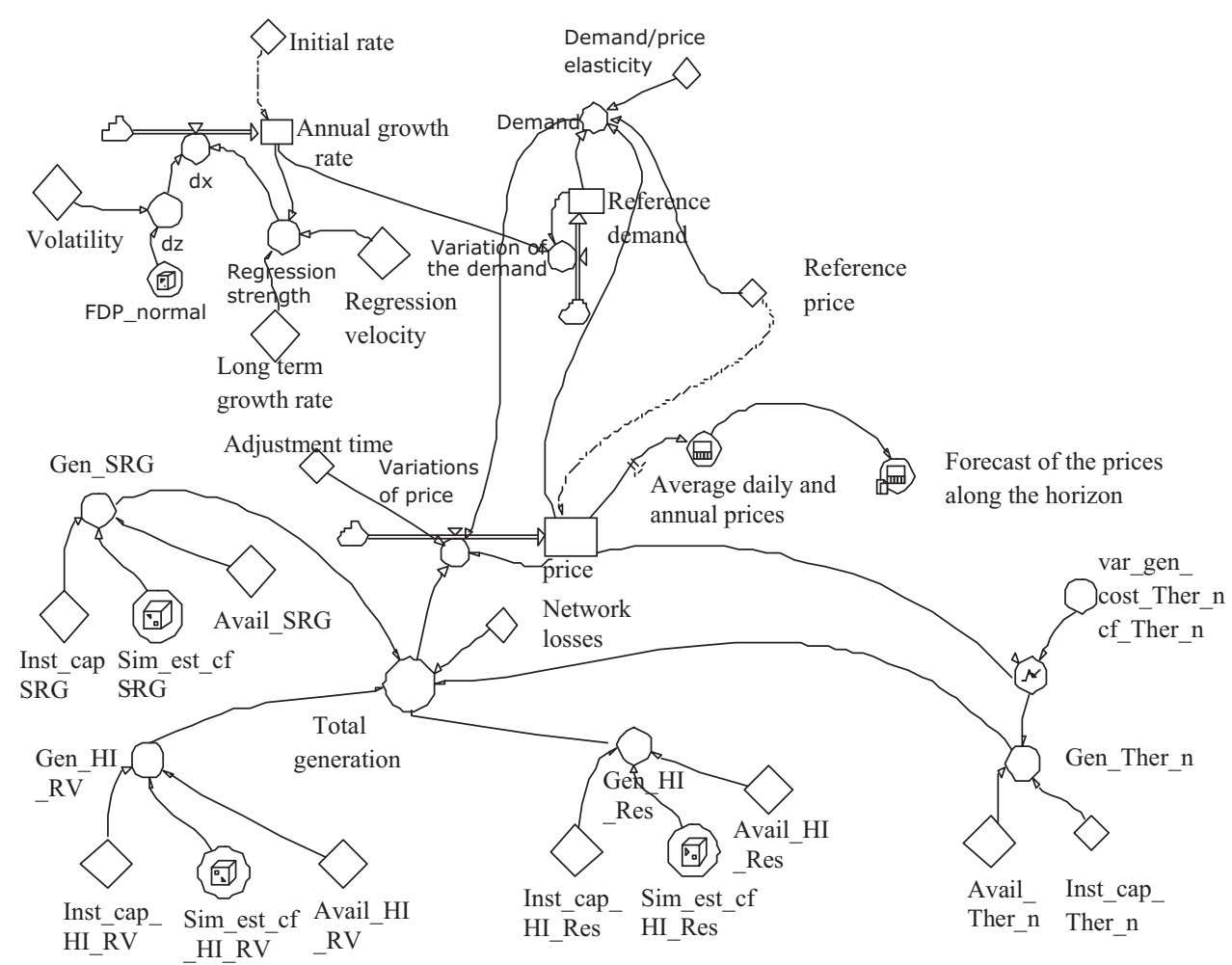

Fig. 4. Dynamic model of the electricity market.

\section{Long term evolution of the power system}

\subsection{Global structure of the model}

The solution of the investment problems detailed in Section 4 requires the knowledge of the evolution of the electricity price and of the capacity factors of each technology. These inputs are provided by the long term model of the electricity market developed using system dynamics. Fig. 4 presents an aggregated view of this model to identify the main sub models and their relations to be detailed in the next sections.

\subsection{The supply system}

The lower part of the diagram of Fig. 4 contains the sub models representing the generation system taking into account their relevant technologies. The left side corresponds to the special regime generation, SRG, the lower central part to the hydro system and the right side to the set of thermal stations.

Regarding SRG, namely the wind parks, we admitted that they are paid feed in tariffs (as it happens for instance in Portugal) or that they can choose between feed in tariffs or receiving the market price plus a premium (as it occurs in Spain). In any case, wind generation has priority over other technologies so that we used historical values for their capacity factor ranging from $20 \%$ to $25 \%$. These values reflect both the availability of the primary resource and of the stations themselves. Using these historical values, in each run of the simulation we sample a value for this index using a stochastic process.

The hydro system is represented by two nodes in Fig. 4 corresponding to run of river, RV, and reservoirs, Res, and, for each of them, we also used historical values of the capacity factor to obtain its average value and standard deviation. Typically, for the Portuguese hydro system, the capacity factor of run of river stations is around $25 \%$ and for reservoirs it ranges from $20 \%$ to $25 \%$ depending on the rainy or dry nature of each year. On the other hand, hydro stations were not subjected to investment decisions in the scope of this problem. Given their reduced operation costs, hydro stations are very attractive and possible locations for new stations have recently been subjected to tenders by official entities. So, we admitted that there is information regarding additions of new hydro additions along the planning horizon coming from these tendering processes.

Finally, Fig. 4 includes a node on the right lower side for thermal stations, Ther. The developed model includes a number of sub nodes according to the existing technologies in the mix. In the case of the Portuguese/Spanish system we modeled combined cycles, coal (eventually further subdivided in different types of coal), fuel and nuclear stations. In this approach we admitted that nuclear is not a candidate technology and that the existing ones are in practice must run stations, typically having a large capacity factor.

The long term dynamic model uses a number of inputs regarding the generation system (installed capacity for each technology, average capacity factors for wind, hydro and nuclear stations) and then it estimates the demand, the electricity price and the capacity factors of the remaining thermal stations. Using the input and the computed capacity factors plus the installed capacity of each technology it is possible to obtain for each period the generation per technology using the specified operation costs. These partial values are then added in the node "Total Generation" and this value is multiplied by a factor larger than 1 to consider network losses, typically in the range from 1.08 to 1.10 .

\subsection{Evolution of the demand}

The demand model is another major block in the dynamic model, given its impact on the electricity price. The demand rate is modeled using an Orneisten-Uhlenbeck mean reverting process [15] given that this model is well suited to incorporate the uncertainty that can affect a variable on the long run [20]. The evolution of the demand 
rate is determined by a long term trend and by a short term component due, for instance, to meteorological conditions. This short term component tends to attenuate so that the demand reverts again to the long term trend. These two terms are included in (9). The first one, $F_{R}$, is given by (10) and it is influenced by the mean value to which the demand rate reverts on the long term, drate ${ }_{L T}$, and by the speed of reversion, $\eta$, modeling how fast variations dissipate and return back to its mean value. The second term is given by (11) and it models the short term component using a Wiener process that depends on the volatility parameter $\delta$. If $\delta$ increases, the demand tends to display larger variations although having the same mean value. Starting at a reference demand rate drate $_{0}$ it is possible to obtain the evolution of the demand rate in the steps of the horizon using (12). In these expressions $\varepsilon_{t}$ represents a random number extracted at each time step from a normal distribution $(0,1)$. This demand model is represented in the upper left part of Fig. 4.

$d x=F_{R}+d z$

$F_{R}=\eta \cdot\left(\right.$ drate $_{L T}-$ drate $\left._{t}\right) \cdot \Delta_{t}$

$d z=\varepsilon_{t} \cdot \delta \cdot \sqrt{\Delta t}$

drate $=$ drate $_{0}+\int_{0}^{T} d x \cdot d t$

Using the demand rate, the model estimates the demand itself using (13). The demand in each period $t$ depends on the demand rate and on the reference demand set for the starting year. Finally, the demand given by (13) can be affected by an elasticity coefficient using (14). In this expression $\pi^{t}, \pi^{0}$ and $E_{d \pi}$ represent the electricity price in period $t$, the electricity price in the starting period and the elasticity of the demand to the price. The value of $E_{d \pi}$ is specified by the planner having in mind that it is typically reduced in current power systems. This block is located in the upper right side of Fig. 4.

$$
\begin{aligned}
& d_{r e f, t}=d_{r e f 0}+\int_{0}^{T} \text { drate }_{t} \cdot d_{r e f 0} \cdot d t \\
& d_{e l e c, t}=d_{r e f, t} \cdot\left(\frac{\pi^{t}}{\pi^{0}}\right)^{E_{d \pi}}
\end{aligned}
$$

\subsection{Evolution of the electricity market price}

Once the electricity demand is obtained for each period $t$, it is possible to estimate the evolution of the electricity price as displayed in the central part of Fig. 4. The price evolution is influenced by the price set at the initial period, $\pi^{0}$, by the demand evolution and by the generation mix, that internalizes the impact of fuel and coal prices together with the availability of wind resources. The price in period $t$ is given by (15) and the price variations $\Delta \pi^{t}$ are given by (16). The price evolution depends on the relation between the demand in period $t$ and the total installed capacity. For instance, if the demand in period $t$ exceeds the installed capacity, than the output of (16) is positive thus inducing a price increase. Conversely, a demand value below the installed capacity will lead to a price reduction. These price variations can be smoothed adopting a value larger than 1.0 for the attenuation factor, $A F$.

$\pi^{t}=\pi^{0}+\int_{0}^{T} \Delta \pi^{t} \cdot d t$
$\Delta \pi^{t}=\pi^{0} \cdot\left(\frac{d_{\text {elec }, t}-G_{\text {total }}}{d_{\text {elec }, t}}\right) \cdot \frac{1}{A F}$

\subsection{Global model and solution algorithm}

Using the dynamic model of the electricity market and the investment planning problems to be solved by each generation company, we can summarize the solution algorithm as follows:

(i) in the first place, the dynamic model is run using the reference demand rate and the generation mix available in the starting period assuming that this mix is unchanged along the planning horizon. This leads to the evolution of the electricity price, of the demand and of the capacity factors of the technologies in the mix.

(ii) using the output of (i), the generation agents solve their profit maximization problems to identify their investment schedules. This means that each generation agent identifies an expansion plan with new capacities to be built, their technologies and commissioning years.

(iii) using the decommissioning schedule of older stations, the set of plans obtained in (ii) and the expected demand evolution, we compute the LOLE and the reserve margin along the horizon and check other global constraints as the ones mentioned in Section 4.2 .

(iv) if no violations are detected in (iii) and if the outputs of step (i) are sufficiently stable along two consecutive iterations, then the iterative process stops. Otherwise, we proceed to step v).

(v) if the problem did not converge yet, then the generation mix is updated using the outputs of step (ii) and the process returns to step (i) to run the dynamic model again.

This approach was implemented in MATLAB, POWERSYM and Microsoft Excel. MATLAB was used to solve the expansion problems detailed in Section 4.1 and to compute the LOLE along the horizon. It was also used an academic version of POWERSIM software package $[21,22]$ to develop the dynamic model using the Runge-Kutta 4th order numerical integration method to solve the differential equations with a time step of $1 \mathrm{~h}$. Finally Excel was used as an interface with the previous two modules and to organize input and output information, as tables and figures.

\section{Case study using data from the Portuguese/Spanish generation system}

\subsection{Initial generation system and data}

The developed approach will now be illustrated using a case study that largely mirrors the Portuguese/Spanish generation system in the scope of the common electricity market involving the two countries. However, it is important to notice that the authors are not in the possession of information regarding the investment strategies of the main players nor of long term forecasts of several parameters that should be used if a more realistic study on the Iberian generation system was to be made. This ultimately means that we used realistic and publicly available data regarding the constitution of the generation systems of both Portugal and Spain and then the remaining required parameters were set using the experience of the authors. The ultimate goal of this study is therefore to illustrate the developed application using a realistic sized system to highlight the main advantages and insights that can be provided by this type of studies.

By the end of 2010 the Portuguese and the Spanish systems had installed capacities of 18,000 and of $99,000 \mathrm{MW}$ and the total demand was 52,204GWh and 260,609 GWh. The transmission 
Table 1

Characteristics of the existing generation mix in the Iberian Peninsula.

\begin{tabular}{lcc}
\hline Technologies & Inst. capacity (MW) & FOR \\
\hline Nuclear & 7777.0 & - \\
Coal_1 & 7500.0 & 0.02 \\
Coal_2 & 5500.0 & 0.02 \\
Fuel/gas turbine & 4500.0 & 0.02 \\
CCGT_1 & $20,000.0$ & 0.02 \\
CCGT_2 & $10,000.0$ & 0.02 \\
Hydro reservoirs & $15,000.0$ & - \\
Hydro run-of-river & $10,000.0$ & - \\
Wind parks & $23,800.0$ & - \\
Photovoltaic & 3500.0 & - \\
Biomass & 800.0 & - \\
Cogeneration & 8600.0 & - \\
\hline
\end{tabular}

systems are well developed and are interconnected by 7 lines at $220 \mathrm{kV}$ and $400 \mathrm{kV}$ and the average market price in 2010 was $55.3 € / \mathrm{MWh}$.

The existing generation mix is described in Table 1. Apart from the respective installed capacities, this table indicates the corresponding FOR, except for nuclear stations, hydro stations, wind parks, photovoltaic, biomass and cogeneration. Regarding nuclear stations, they typically cover the basis of the diagram and the energy provided by them was obtained using historic data that already reflects outages and preventive maintenance. As detailed in Section 5.2 wind parks, photovoltaic, biomass and cogeneration plants correspond to special regime stations both in Portugal and in Spain. For operation purposes, these special regime technologies can typically inject in the networks as much energy as available given the volatile nature of the primary resource used by some of them. For modeling purposes we also used historic data indicating the energy provided by these technologies in recent years and deriving from these values the corresponding capacity factors. These values reflect both the availability of the primary resource and the availability of the generation stations themselves.

The simulation was run considering 7 generation companies, Genco_1 to Genco_7, that own the mentioned installed capacity as follows: Genco_1 has $16.5 \%$, Genco_2 has $22.0 \%$, Genco_3 has $5.2 \%$, Genco_4 has $12.6 \%$, Genco_5 has 3.7\%, Genco_6 has $10.0 \%$ and Genco_7 has 30\%. Genco_7 represents the aggregated share of a large number of small generation agents. Further assumptions to model the generation system are as follows:

- regarding the nuclear stations, we admitted a capacity factor of $85 \%$ with a standard deviation of $5 \%$;

- the operation of the remaining thermal stations depends on their operation costs, on the evolution of the electricity price and of the demand along the simulation. In order to model in a more refined way the technologies in the generation mix, we divided coal and CCGT stations in two sets each, enabling specifying different costs for each class;

- hydro stations were also divided in two sets - reservoirs and run of river stations. In the simulation, we used an average capacity factor of $20 \%$ for reservoirs and $25 \%$ for run of river together with a standard deviation of $5 \%$;

- for the wind parks and photovoltaic systems we admitted average capacity factors of $25 \%$ and of $20 \%$ based on historic values and a standard deviation of $5 \%$;

- finally for cogeneration and biomass systems we used average capacity factors of $45 \%$ and of $55 \%$.

The planning horizon was set at 15 years and we admitted there are a number of stations to be decommissioned as follows: $1500 \mathrm{MW}$ of nuclear groups in year 3, for Coal_1 $1000 \mathrm{MW}$ in year 4 and $1000 \mathrm{MW}$ in year 8 and finally $1000 \mathrm{MW}$ of CCGT_1 in year 10 . Given the nature of the special regime technologies, there are in the two countries special licensing schemes, namely using tender mechanisms for new wind capacity. Therefore, we admitted there will be new wind capacity as follows: $200 \mathrm{MW}$ per year from year 1 to 4 and $100 \mathrm{MW}$ from year 5 to year 10 . For photovoltaic systems we admitted $200 \mathrm{MW}$ in year 3, $150 \mathrm{MW}$ in year 6 and $200 \mathrm{MW}$ in year 9. Finally, new hydro capacity has also been subjected to a tender process in Portugal and so we admitted $500 \mathrm{MW}$ of new hydro capacity in year 6 and $300 \mathrm{MW}$ in year 10 .

The demand in the departing year of the horizon was set at $312.8 \mathrm{TWh}$. The yearly demand was modeled using a load duration curve with 6 steps as follows: $100 \%$ of the peak power during $5 \%$ of the year, $90 \%$ for $20 \%$ of the year, $80 \%$ for $45 \%$ of the year, $70 \%$ for $65 \%$ of the year, $60 \%$ for $85 \%$ of the year and $50 \%$ of the peak power during $100 \%$. The long term demand rate was set at $2 \%$ and then along the planning horizon the demand rate is adjusted by the dynamic model but the above discretization of the load diagram remains unchanged.

As for the technologies used in the expansion planning problems, we considered three options as indicated in Table 2. We also assumed that the life time of these stations is 30 years and that the construction period is 3 years for Techs_ 1 and 3 and 2 years for Tech $\_$. The operation costs were modeled using a cost function organized in five segments and established in function of the capacity factor. As an example, for Tech_1 we specified the following points $(0.0 ; 0.0),(0.2 ; 15.0),(0.4 ; 30.0),(0.6 ; 40.0),(0.8 ; 65.0)$ and $(1.0 ; 80.0)$. In each of these pairs, the first element represents the capacity factor and the second is the variable operation cost in $€ /$ MWh.

In order to prevent market power and to incorporate energy policy objectives we also admitted that in each year of the horizon each agent cannot install more than $800 \mathrm{MW}$ in Tech_1, $400 \mathrm{MW}$ in Tech_2 and $400 \mathrm{MW}$ in Tech_3 and the total new capacity in any of these technologies should not exceed $50 \%$. As indicated in the beginning of this section these limits were adopted by the authors just for illustration purposes and have no direct connection with the investment policies of the generation players in the system. However, some examples of such limitations were already put in practice in the recent past. For instance, in recent years the Portuguese government used public auctions to allocate new hydro capacity and the current largest Portuguese generation company was forced to reduce its generation assets in order to be allowed to build new hydro capacity. As another example, in 2008 it was opened an auction to allocate new CCGT stations and among the rules set for this auction there were limitations regarding the total amount of power that could be allocated to the same agent. The simulations used a number of other input parameters as follows: the yearly reserve margin should be larger than $30 \%$, the maximum value allowed for LOLE is $2 \mathrm{~h} / \mathrm{year}$, and a $7 \%$ actualization rate was used along the planning horizon to bring revenues and costs to the initial period.

Once again to illustrate the capabilities of the developed application, we admitted that Genco's 5, 6 and 7 are not interested in expanding their generation capacity. On the other hand, Genco's 1-4 are available to invest in these three technologies considering the following financial limitations:

- Genco_1 has $1200 \mathrm{M} €$ available for the period from year 1 to year 10 plus $400 \mathrm{M} €$ in the final 5 years;

- Genco_2 has $500 \mathrm{M} €$ available for the first 5 years, plus $1200 \mathrm{M} €$ for the final 10 years;

- Genco_3 has $500 \mathrm{M} €$ from year 1 to 5 , plus $500 \mathrm{M} €$ from year 6 to 10 , plus $500 \mathrm{M} €$ for the final 5 years;

- Genco_4 has no resources to invest from year 1 to 3 . From year 4 till year 15 it has $1200 \mathrm{M} €$ available. 
Table 2

Characterization of the candidate technologies.

\begin{tabular}{|c|c|c|c|c|c|}
\hline Type of technology & Available capacities (MW) & Investment cost (€/MW) & Fix $O \& M$ cost $(€ / M W$ year $)$ & Cost of capital with the loan ( $€ /$ MW year) & FOR \\
\hline Tech_1 & 200 or 400 & 650,000 & 7050 & $52,380.40$ & 0.02 \\
\hline Tech_2 & 200 or 300 or 400 & 500,000 & 6200 & $40,230.40$ & 0.02 \\
\hline Tech_3 & 200 or 400 & $1,050,000$ & 8600 & $82,300.10$ & 0.02 \\
\hline
\end{tabular}

Table 3

Generation expansion plan obtained for Genco_1.

\begin{tabular}{lccc}
\hline Stage & Tech_1 $(\mathrm{MW})$ & Tech_2 $(\mathrm{MW})$ & Tech_3 $(\mathrm{MW})$ \\
\hline 2 & 400 & - & - \\
3 & 200 & 200 & - \\
5 & 400 & - & - \\
6 & - & 200 & 200 \\
9 & - & 200 & - \\
12 & 200 & - & 200 \\
\hline
\end{tabular}

Table 4

Generation expansion plan obtained for Genco_2.

\begin{tabular}{lccc}
\hline Stage & Tech_1 (MW) & Tech_2 (MW) & Tech_3 (MW) \\
\hline 2 & 400 & - & - \\
3 & - & 200 & - \\
6 & 400 & 200 & 200 \\
10 & - & 200 & - \\
11 & 200 & 200 & 200 \\
\hline
\end{tabular}

Table 5

Generation expansion plan obtained for Genco_3.

\begin{tabular}{lccc}
\hline Stage & Tech_1 (MW) & Tech_2 (MW) & Tech_3 (MW) \\
\hline 3 & 400 & - & - \\
4 & - & 200 & 200 \\
6 & 400 & - & - \\
7 & - & 200 & - \\
11 & 200 & - & 200 \\
\hline
\end{tabular}

\subsection{Results of the generation expansion study - base case}

In the first place, the approach described in Sections 3-5 was used to develop the expansion plans of Genco's_1-4 using the above data and admitting that the capacity payment incorporated in (2) is set to zero. Tables 3-6 detail the obtained four expansion plans indicating the year in which investment decisions are taken. This means, for instance that Genco_1 decided to build a $400 \mathrm{MW}$ station of Tech_1 in year 2 but this station will only start operation at year 5 , given its construction time of 3 years.

Regarding the new additions, $50 \%$ of the new installed capacity is for Tech_1, 28.94\% for Tech_2 and $21.06 \%$ for Tech_3. Tech_1 is the most competitive one and its share is not even larger because it was imposed that the total new capacity in any technology should not exceed $50 \%$. The distribution of the new capacity by the four Genco's is as follows: $30.26 \%$ for Genco_1, 28.95\% for Genco_2, 22.36\% for Genco_3 and $18.43 \%$ for Genco_4. The maximum value of LOLE occurred in year 7 , reaching $0.88 \mathrm{~h} /$ year. Fig. 5 displays the evolution of the yearly average electricity price estimated by the dynamic model. In the beginning, the average price tends to increase because the first new stations will only start operation at year 5 , taking into account the 3 year construction period. The price does not rise even

Table 6

Generation expansion plan obtained for Genco_4.

\begin{tabular}{lccc}
\hline Stage & Tech_1 (MW) & Tech_2 (MW) & Tech_3 (MW) \\
\hline 5 & 400 & 200 & 200 \\
10 & 200 & - & 200 \\
11 & - & 200 & - \\
\hline
\end{tabular}

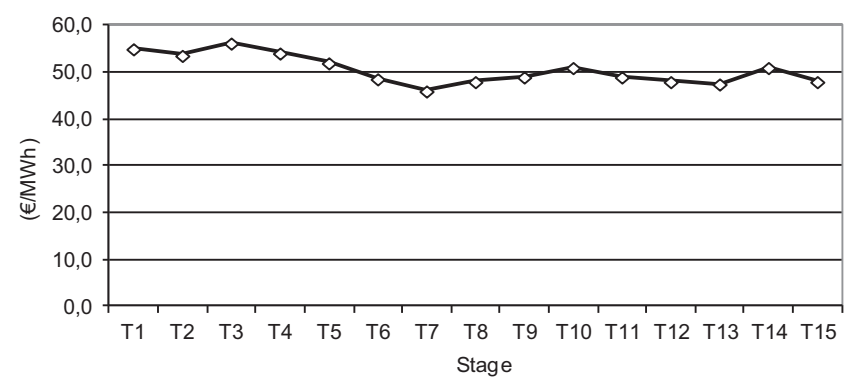

Fig. 5. Evolution of the average electricity price along the planning horizon.

more because there are new additions of wind parks and photovoltaic systems in the first years of the horizon. The peak in year 3 is also determined by the decommissioning of $1500 \mathrm{MW}$ of nuclear groups.

Finally, Fig. 6 displays the evolution of the capacity factors of the three candidate technologies. These values are a result of the operation cost of each technology, together with the evolution of the demand, the decommissioning of existing stations and the use of hydro stations and the special regime stations. For Tech_1 the graph starts in year 5 given the year in which the first decisions are taken and the construction period. For similar reasons, the graphs for Tech_2 and 3 start in year 5 and in year 7 respectively. In average, Tech_1 has the largest capacity factor, displaying an average value of 0.69 per year, followed by Tech_3 (average value of 0.54 per year) and by Tech_2 (average value of 0.52 per year).

\subsection{Sensitivity analysis - impact of $\mathrm{CO}_{2}$ emission costs}

For illustration purposes, we also admitted a scenario in which $\mathrm{CO} 2$ emission costs were internalized in the model. We admitted that Tech_1 emits $0.77 \mathrm{CO}_{2}$ ton/MWh, Tech_2 emits $0.36 \mathrm{CO}_{2}$ ton/MWh and for Tech_3 we used $0.09 \mathrm{CO}_{2}$ ton/MWh reflecting the use of cleaner technologies in the case of Tech_3. On the other hand, we used a cost of $20.0 € /$ ton of $\mathrm{CO}_{2}$, considering prices on $\mathrm{CO} 2$ emission markets, and the capacity term in (2) was not used. Using these values, Tech_2 and Tech_3 become more attractive regarding the base case. As a result of the $\mathrm{CO}_{2}$ emission costs, the average electricity price increased from $51.1 € / \mathrm{MWh}$ in the base case to $58.6 € / M W h$. In this new scenario the new capacity additions are as follows: $33.20 \%$ for Tech_1, 26.80\% for Tech_2 and $40.00 \%$ for Tech_3. Regarding the evolution of the capacity factors, the average value obtained for Tech_1 decreased from 0.69 to 0.52 per year while for

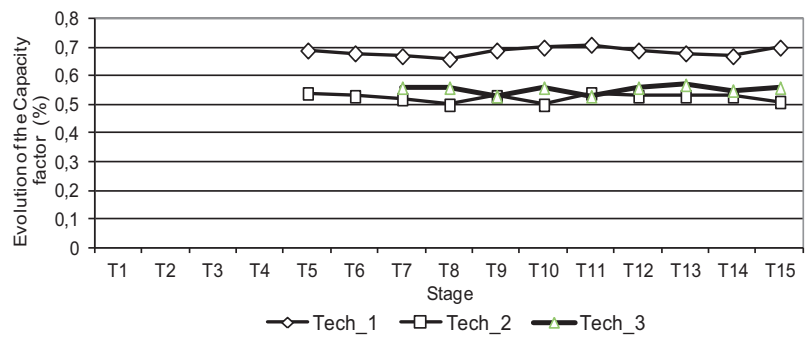

Fig. 6. Evolution of the capacity factor of the three candidate technologies. 
Tech's 2 and 3 they increased from 0.52 to 0.57 and from 0.54 to 0.71 .

This type of studies shows that changes in legal, tariff or regulatory provisions can originate significant alterations on the investment plans confirming that the generation activity is now more risky than in the past.

\subsection{Sensitivity analysis - impact of increasing wind parks by $20 \%$}

The generation system of both countries already has a remarkable penetration of renewable stations, namely hydro and wind parks. In order to get insight on the eventual impact of a larger increase of the installed capacity in wind parks, we admitted that the installed capacity in the initial year was increased from $23,800 \mathrm{MW}$ as indicated in Table 1 to $28,560 \mathrm{MW}$, that is, a $20 \%$ increase. Once again the capacity term in (2) was not used. As a result of this wind capacity increase, the installed capacity in the three candidate technologies got reduced when compared with the values reported in Section 6.2. Regarding Tech_1 the installed capacity reduced from $3800 \mathrm{MW}$ to $3200 \mathrm{MW}$, for Tech_2 it reduced from $2200 \mathrm{MW}$ to $1900 \mathrm{MW}$ and for Tech_3 it reduced from $1600 \mathrm{MW}$ to $1400 \mathrm{MW}$. This means there is a reduction of the installed capacity in the three candidate technologies of $14.5 \%$. On the other hand, the average value of the electricity price along the planning horizon got reduced from $51.1 € / \mathrm{MWh}$ in the base case to $48.9 € / \mathrm{MWh}$, that is a reduction of $4.3 \%$. It is important to notice that this market price reduction does not necessarily mean that the final end users will also see a price reduction. In fact, in Portugal for instance renewable stations are paid feed-in tariffs that currently have an average value of $70.0 € / M W h$ that will in any case be incorporated in the final end user tariffs. A direct consequence of this increased amount of renewable installed capacity is the progressive reduction of the income of generation companies having thermal stations, not only because the market price gets reduced but also because the number of hours these stations will be used in each year also gets reduced.

\subsection{Sensitivity analysis - impact of the implementation of a capacity payment term}

As mentioned in Section 6.4, having a large amount of installed capacity using renewable resources has important consequences for the remaining generation agents, namely for the ones owning traditional thermal stations. In fact, the market price tends to get reduced either because new generation bids at zero price are incorporated in the aggregated market selling curves or because the liquid demand to be supplied by traditional stations gets reduced. In both cases, the result is that the intersection of the aggregated buying and selling curves occurs for more reduced market prices. Apart from that, the number of operating hours along each year of several thermal stations tends to get reduced, that is, the capacity factors of several stations are reduced. This effect is already visible in several stations both in Portugal and in Spain, where some recently built CCGT stations operate less than $2000 \mathrm{~h}$ a year, thus compromising their profitability.

Having in mind these facts, we started by computing the average return rate associated with the thermal stations for the investment plans obtained in the base case described in Section 6.2 and also when admitting that the installed wind power capacity increased by $20 \%$, as described in Section 6.4. This return rate was computed as the quotient of the profit associated to a specific station regarding its total investment and operation costs along the entire planning horizon. On the other hand the profit corresponds to the difference between the income obtained from selling the electricity at the market prices and the total investment and operation costs.
Using this approach, for the base case we obtained an average return of $11.3 \%$ and this value gets reduced to $9.6 \%$ when considering the increase of $20 \%$ of the wind power installed capacity. This reduction can freeze the investment decisions in new thermal capacity or can ultimately originate the shut down of some already existing thermal stations, as it is apparently under consideration by some Portuguese generation agents, thus eventually compromising in the long term the security of supply.

In other to counteract this impact of renewable installed capacity, we used the developed GEP model to estimate the value of the capacity payment term included in (2), $P_{\text {cap }}^{j}$, that should be considered in order to obtain the $11.3 \%$ return rate of Section 6.2, but using the expansion plans identified in Section 6.4 when admitting that the wind power capacity increased by $20 \%$. After running the GEP model several times using different values for the capacity payment term, we estimated a value of $5.6 € / \mathrm{MW}$ for the capacity term in order to guarantee the $11.3 \%$ rate of return.

This type of studies can be used in a profitable way both by state or regulatory agencies to get more insight on the impact of adopting different values for $P_{\text {cap }}^{j}$. For instance, the entire expansion planning model including the long term dynamic simulation was run using a capacity payment value of $10.0 € / \mathrm{MW}$. In this case, the investment plans would now include the installation of $3400 \mathrm{MW}$ of Tech_1 (more $200 \mathrm{MW}$ regarding the solution described in Section 6.4), $2100 \mathrm{MW}$ of Tech_2 (more $200 \mathrm{MW}$ than the solution in Section 6.4) and 1400 MW of Tech_3 (same value as obtained in Section 6.4). Given that the installed capacity is now slightly increased, the capacity factors of the thermal stations get slightly reduced and, as a result, the return rate along the planning horizon gets now reduced from $11.3 \%$ to $10.8 \%$. However, this solution is advantageous regarding the immediate incorporation of a $5.6 € / \mathrm{MW}$ payment using the same plans as obtained in Section 6.4 because the total installed thermal capacity becomes larger thus increasing the security of supply in face of the large presence of generation using volatile primary resources.

\section{Conclusions}

This paper describes a long term approach to the generation expansion planning problem combining the solution of expansion profit maximization problems by individual generation agents together with a long term dynamic simulation to estimate the evolution of the demand and of the electricity price. The developed approach was illustrated using a generation system that incorporates the most relevant characteristics of the Portuguese/Spanish generation system. The developed approach can be used in a profitable way by generation companies to help them preparing their own expansion plans, simulating possible behaviors of other competitors, as well as to run sensitivity studies to investigate the impact, for instance, of changes on the operation or investment costs or on the long term evolution of the demand. It can also be used by regulatory or state agencies to evaluate the long term impact regarding market or regulatory design changes, so that more sounded and less risky options are adopted. As an example, the developed approach can be used to evaluate the impact of introducing a capacity term to remunerate stations that are becoming less used given the massive deployment of dispersed generation, namely wind parks and solar systems, in Portugal and Spain. As a result of these large investments, the capacity factors of several thermal stations, including recently built CCGT's, are very reduced thus eventually justifying the introduction of such capacity term. The developed approach can be used to investigate the impact of such terms and to help regulatory and state agents to adequately set and calibrate their values. In brief, this paper is an attempt to look more closely on recent years and to anticipate what may 
happen in several power systems till 2030, taking as a realistic example the generation systems of Portugal and Spain.

\section{References}

[1] B.G. Gorenstin, N.M. Campodonico, J.P. Costa, M.V. Pereira, Power system expansion planning under uncertainty, IEEE Transactions on Power Systems 8 (1993) 129-136.

[2] B.F. Hobbs, Optimization methods for electric utility resource planning, European Journal of Operational Research 83 (1995) 1-20.

[3] K. Vlahos, Capacity planning in the electricity supply industry, Ph.D. Dissertation, London Business School, London, 1990

[4] J. Sirikum, A. Techanitisawad, V. Kachitvichyanukul, A new efficient GABenders' decomposition method: for power generation expansion planning with emission controls, IEEE Transactions on Power Systems 22 (2007) $1092-1100$.

[5] J.C. Meza, M.B. Yildrim, A.S. Masud, A model for the multiperiod multiobjective power generation expansion planning, IEEE Transactions on Power Systems 22 (2007) 871-878.

[6] C.H. Antunes, A.G. Martins, I.S. Brito, A multiple objective mixed integer linear programming model for power generation expansion planning, Energy 29 (2004) 613-627.

[7] J.-B. Park, Y.-M. Park, J.-R. Won, K.Y. Lee, An improved genetic algorithm for generation expansion planning, IEEE Transactions on Power Systems 15 (2000) 916-922.

[8] F. Wu, Z. Yen, Y. Hou, Y. Ni, Applications of AI Techniques to Generation Planning and Investment, in: Proceedings of the IEEE PES General Meeting, Denver, USA, vol. 1, 2004, pp. 936-940.

[9] J.J. Zhu, M. Chow, A review of emerging techniques on generation expansion planning, IEEE Transactions on Power Systems 12 (1997) 1722-1728.

[10] M. Yildirim, K. Erkan, S. Ozturk, Power generation expansion planning with adaptive simulated annealing genetic algorithm, International Journal of Energy Research 30 (2006) 1188-1199.

[11] J.-B. Park, J.-H. Kim, K.Y. Lee, Generation expansion planning in a competitive environment using a genetic algorithm, in: Proceedings of the IEEE PES Summer Meeting, Chicago, USA, vol. 3, 2002, pp. 1169-1172.

[12] A. Botterud, M. Ilic, I. Wangensteen, Optimal investment in power generation under centralised and decentralised decision making, IEEE Transactions on Power Systems 20 (2005) 254-263.
[13] J. Forrester, System dynamics and the lessons of 35 years, in: K.B. De Greene (Ed.), The Systemic Basics of Policy Making in the 1990, Sloan School of Management, MIT, Boston, USA, April 1991, pp. 1-35.

[14] A. Ford, System dynamics and the electric power industry, System Dynamics Review 3 (1997) 57-85.

[15] F. Olsina, F. Garces, H.-J. Haubrich, Modelling long-term dynamics of electricity markets, Energy Policy 34 (2006) 1411-1433.

[16] T. Kadoya, T. Sasaki, S. Ihara, E. Larose, M. Sanford, A.K. Graham, C.A. Stephens C.K. Eubanks, Utilizing system dynamics modelling to examine impact of deregulation on generation capacity growth, Proceedings of the IEEE 93 (2005) 2060-2069.

[17] T.S. Jalal, P. Bodger, The development of a system dynamics model to evaluate generation expansion in New Zealand, in: Proceedings of AUPEC 2010, Australasian Universities Power Engineering Conference, Christchurch, New Zealand, 2010, pp. 1-6.

[18] A.J. Pereira, J.T. Saraiva, A decision support system for generation expansion planning in competitive electricity markets, Electric Power Systems Review 80 (2010) 778-787.

[19] A.J. Pereira, J.T. Saraiva, Generation expansion planning - a long-term approach using system dynamics and genetic algorithms, Energy 36 (2011) 5180-5199.

[20] A. Dixit, R. Pindyck, Investment Under Uncertainty, Princeton University Press, New Jersey, USA, 1994.

[21] Powersim Software AS, Powersim 2.5 User's Guide and Reference Manual, Powersim Press, Bergen, Norway, 1996.

[22] Powersim Software AS, Powersim Studio 7 Academic, Powersim Software AS Bergen, Norway, 2006.

Adelino J.C. Pereira was born in Sanfins, Portugal in 1975. He received his diploma and M.Sc. degrees in Electrical Engineering and Computers from the Faculdade de Engenharia da Universidade do Porto, FEUP, Portugal, in 1998 and 2003. In 1998 he joined the Coimbra Polytechnic Institute (ISEC) where he is currently Adjunct Professor. In July 2010 he concluded his Ph.D. at FEUP with a Thesis on long term generation expansion planning using system dynamics.

João Tomé Saraiva was born in Porto, Portugal in 1962. In 1987, 1993 and 2002 he got his M.Sc., Ph.D., and Agregado degrees in Electrical and Computer Engineering from the Faculdade de Engenharia da Universidade do Porto where he is currently Professor. In 1985 he joined INESC Porto where he was head researcher or collaborated in several projects related with the development of DMS systems, quality in power systems, and tariffs due for the use of transmission and distribution networks. Severa of these projects were developed under consultancy contracts with the Portuguese Electricity Regulatory Agency. 